\title{
La investigación sobre las necesidades de información en comunidades de usuarios
}

\author{
Juan José Calva González \\ CentroUniversitariodeInvestigacionesBibliotecológicas \\ de la UNAM, 04510, Mé xi coD.F., Tel: 56-23-03-38 \\ E-mail:jjcg@servidor.unam.mx
}

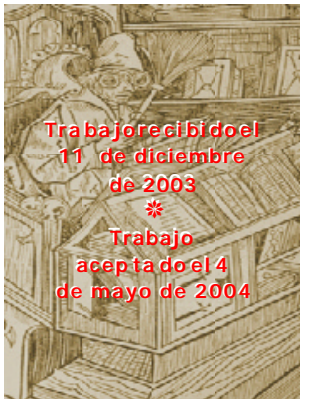

\begin{abstract}
RESUMEN
El documento expone lo relativo a diversas investigaciones realizadas sobre el fenómeno de las necesidades de información en varias comunidades de usua rios delain forma ción. Asi mis mo mues tralas más im por tan tes y que mayor tras cen den cia han te ni do en lo re la ti vo a ser el pun to de par ti da de otras investigaciones. Tambiénseanalizanestasinvestigaciones conlafinalidadde ex traery con cluir a par tir de ellas diver sas si militu des con res pec to al es tu dio del fe nó me no y de en con trar las di fe ren cias exis ten tes se gún los ti pos de comu ni da des de usua rios, a la vez que se ex po nen los fac to res ex ter nos ein ternos que ofrecen las diferencias y similitudes. Se toman en consideración las fases del fenómeno expuestas siguiendo el Modelo sobrelas necesidades de información (Modelo NEIN).
\end{abstract}

Pa la bras cla ve: Necesida des de in for ma ción, Mo de lo nein, Comu ni da des de usua rios.

\section{RESEARCH ON INFORMATION NEEDS OF USER COMMUNITIES JuAN JosÉ CALVA-GONZÁLEZ}

\section{RESUMEN}

The pa per deals with stud ies made on the in for ma tion needs in sev eral commu ni ties of in for ma tion us ers. It also ap proaches the com mu ni ties that have shown more importance and have become the start for other researches. Simi lari ties and dif fer ences are ana lyzed in these us ers ac cord ing to the type of communities. The different phases of this phenomenon are exposed following the NEIN Model on information needs.

Keywords: In for mations needs, NEIN Model, Commu ni ties of users. 


\section{INTRODUCCIÓN}

$\mathbf{L}$ os seres humanos desde sus albores han tenido la idea de conservar el conocimiento, primero de forma oral y después, con la invención de la escritura, de plasmarlo en un soporte que pudiera ser consultado por las generaciones futuras, comolo fuela pie dra, el pa pi ro, el perga mi no, ellibro y ahora el me dio elec tró ni co.

El acceso y consulta de la información registrada en cualquier soporte abrió las pues tas a la exis ten cia de la ins ti tu ción so ciallla ma da biblio teca, la cual se ría la encargada no sólo de conservar la información registrada sino de hacerla accesible a una comunidaddesujetosousuarios.

Ahora bien, existen comunidades de usuarios formadas por diferentes tipos de suje tos pero con ca rac te rís ti cas que los ha cen co mu nes, como por ejem plo los ado les centes de 12 a 15 años. Comolas co mu ni da des es tán com pues tas porpersonas entonces tienen necesidades de información; es decir, pasan por el proceso de surgimiento de una necesidad informativa que los conduce a un comportamiento en la búsquedadein formaciónconelfindesatisfacerdichanecesidad.

Las biblio te cas ylas co mu ni da des de usua rios quelas utilizan han sidolos objetos de es tu dio deva rias inves tiga cio nes, prime ro des de el usua rio real y des pués to mando en cuenta al potencial, para de ahí estudiar a diversas comunidades no ligadas a una unidad deinformación sino simplemente con sidera das comouna co mu nidad de usua rios dela in for ma ción y es tu diar el fe nó me no ahípre ci sa men te, y des cu brir re cursos informativosdiferentes al uso de la biblioteca para satisfacernecesidades de informacióndecomunidadesespecíficas.

A partirdeeste su pues to es precisoplan tearlas siguien tes pre gun tas:¿quéin ves tigaciones se ha realizado en América Latina, España y, principalmente, en México acerca de este fenómeno de las necesidades de información? ¿qué trabajos se han presentadoenlas Jorna das Mexica nas de Biblio te conomía? ¿quéfase (o fases) delfenómenodelas necesidades deinforma ciónsehaninvestigado? ¿existeninvestigaciones con diversas po bla cio nes de usuarios? Es tas pre gun tas son las queim pulsa ron la ela boración deeste documen to, elcual tienecomo finalidad presentaruna selección detrabajos quepermitancon tarcon unacercamien to deloque acon te ce en la re gión yalavezteneruna respuestaaproximadaalaspreguntasantesplanteadas.

\section{EL EST UDIO DEL USUARIO Y SUS NECESIDADESDE INFORMACIÓN}

Por muchos años las investigaciones estuvieron enfocadas principalmente a los usua rios reales de jando de lado a los po ten ciales, ${ }^{1}$ esto quie re de cir que siem pre se ha

1 J.M. Brittain, "Pitfall of user orien ted re search and some ne glec ted areas", en Intemationalrearch formininformationsience 1981, vol.. 4, pp. 213-227. 
vis to al usua rio como aquel que en tra en unaunidad dein for ma cióny deman da al gún servicio oin formación y seolvida que to doslosin dividuos tienen necesida des deinformaciónyquelasbibliotecas existenparaatendersusnecesidadesysatisfacerlas.

Aun que esto es así, McDiar mid ${ }^{2}$ en un do cu men to del año de 1940, ya se cues tionaba en tor no ala exis ten cia degrupos de usuarios dela in for ma ción quenoacu díana utilizarlabiblio te ca; en trelas preguntas que po de mos de rivarde estedo cumen to se en cuen tran las siguien tes:¿quiénes sonlos usua rios poten cia les? ¿cuáles son los intereses y nece si da des de in forma ción delos usua rios po ten ciales? ¿cuáles son sus ac titudescon relaciónalabibliotecaquelosatiende?

Sin embargo, esta preocupación por los usuarios que no asisten o acuden a una unidadde in formación se originó aproxima da mente a me dia dos delsiglo XX, épo ca en la que se inicia la importancia de abordar las necesidades de información de las perso nas que no asis ten a di chas uni dades. Debe to marse en cuen taque du ran te mu chos años la preo cu pa ción de las biblio te cas se cen trabaen orga nizarel mate rial y no en elsujetoousuarioqueera eldestinata riofinaldeesematerialqueseorganizaba.

Perocuandolabalanzasedesplazahacia el sujetoousua rioquenecesitayusa rálainforma ción es cuan do se pien sa enlas nece si da des dein formación, las cuales no sólo se conocen conunasimplepregunta realizadadu ran telainves tiga ciónoes tu dio deusuarios y que se ría: ¿cuáles son sus ne ce si da des de in for ma ción? ${ }^{33} \mathrm{La}$ cual, en un principio está ex clu siva men tedirigidaalos usua rios realesin tegran tes deun sec tor de la comunidady sóloconel tiem po pasa ha cialos poten cia les para conocer también cuáles son sus necesidades y de termi nar elgra do de coin ci den cia en tre unos yotros. ${ }^{4}$ Por ello el proble ma, más fá cil de en fren tar y de re solver es que para las uni da des dein for ma ción es mássencilloestudiaralosusuariosrealesquealosusuariospotenciales. ${ }^{5}$

Lo an te rior es así, ya que de for ma con ven cio nal o tra di cio nallos servi cios de información son dirigidos a los usuarios reales, quienes expresan sus necesidades vía una demanda en el servicio bibliotecario y de información, pero tampoco aquí se con templan oinves tiganlas nece si da des de in for ma ción queno son convertidasen esas demandas ${ }^{6}$ poreste tipo de usuarios reales; asi mis molos servicios seolvidan por comple to delos usua rios po tencia les que en reali dad son un porcentajema yo rita rio delacomunidaddeusuariosquenormalmenteatiendeunaunidaddein formación.

Porotroladoelusua rio en mu chasocasiones noescapazde ex pre sarloque ne ce si ta pues a ve ces los usua rios no sa ben en for ma pre ci sa lo que quie ren y es el bibliotecario

2 E. W. Mcdiarmid. Thelibrarysurvey: problensandmothods Chi ca go, ALA, 1940.

3 H. Men zel. "The in for ma tion ne eds of cu rrent scien ti fic re search" en Libraryquately, 1964, vol. 34 , p. 15

4 Elias Sánz Casado. Manual deestudios deusuanios Madrid: Fundación GermánSánchezRuipérez, 1994, p. 29.

5 N.M. Fi guei re do. Estudbsdusoeusuariosdainformaçaa Bra silia:Ins titu to Brasileirode In formacao em Cien cia e Tec no lo gia, 1994, p.42.

$6 \quad$ Idam 


\section{InvestigacónBiblicteedógica v. 18 N o. 37 julio/ diciembre de 2004}

quien tienequecon se guirqueel usua rioverbalicesus nece sida des dein forma ción de una formaclarayunívoca. ${ }^{7}$

Losobjetivosdelasinvestigacionesrealizadas so breelfenó me no delas necesidades de información y el comportamiento informativo pueden ser diversos, y pocos reúnen en un solo trabajo la totalidad de aspectos imbricados en el fenómeno que ocupaestedocumento, comolos siguientes:

a) La explicación del fenómeno de expresión de las necesidades de información.

b) El conocimiento de las necesidades de información que tiene una comunidad determinada.

c) La predicción de las necesidades de información de un grupo de sujetos.

d) La determinación del comportamiento informativo de la comunidad de sujetos.

e) La predicción del comportamiento informativo

f) El nivel de satisfacción de las necesidades de información de un grupo de sujetos que integran una comunidad

g) El control de la información que será utilizada para satisfacer las necesidades de información.

Elconocimien tosobrelasnecesidades dein formacióndeunacomunidadimplica tener las basesprima rias de to daslasactividadesin formativas ${ }^{8}$ de los sujetos que la forman conla finali dad de que di cho conocimien to sea utiliza do porlas diversas uni dades para el desarrollo de colecciones, de los servicios y en general de la propia unidadinformativa.

Pero frecuentemen telos resultados de es tu dios empíricos sobrelos te mas denecesidades deinformación y comportamien toin formativo, han sidoúnicamenteacu mulacióndedatos conocidos previamen teyllevados aconfirmartodaslas relaciones conocidas, peronohangeneradoconocimientonuevo, ${ }^{9}$ que es jus to lo que se re quierepara elde sarro lloy avan ce dela biblio te cología en cuan to a usua rios, colec ciones y servicios.

SegúnelModelosobrelasnecesidadesdein forma ción(Modelo NEIN) ${ }^{10}$ el fe nómeno de las necesidades de información consta de tres fases principales: el surgimien to dela nece sidad dein forma ción, el com porta mien to enla bús que da dein formación y la satisfacción a dichas necesidades. El análisis de las investigaciones y estudios de usuarios llevados a cabo en diversas comunidades de usuarios abordan

7 E. Sánz Casados. Op at., p. 24.

8 N.H. Prasad. Infomation need and user Va ra na si: In dian Bi blio gra phic Cen ter, 1992,p 26.

9 W. H. Kunz, W.J. Ri ttel y W. Schwu chow. Methoosofanalysisandevaluationofinformationneets Munchen : Verlag Dokumentation, 1977, p. 10.

10 CfrJuan José Cal va Gon zá lez.Análisisdlasnecesidadesdeinformacóndbamental. Teońaymátodbs Madrid: JJCG, 2001. Tesis (Doctor en Ciencias de la Información)—Universidad Complutense de Madrid.

También se en cuen tra en pren sa la des crip ción del Mo deloNEIN en el libro: Calva Gon zález,Juan José. Lasnecesidadesdeinformađaón Fundamentosteónicosymátodos Mé xi co:UNAM, Cen tro Universita rio Investigaciones Bibliotecológica, 2004. 


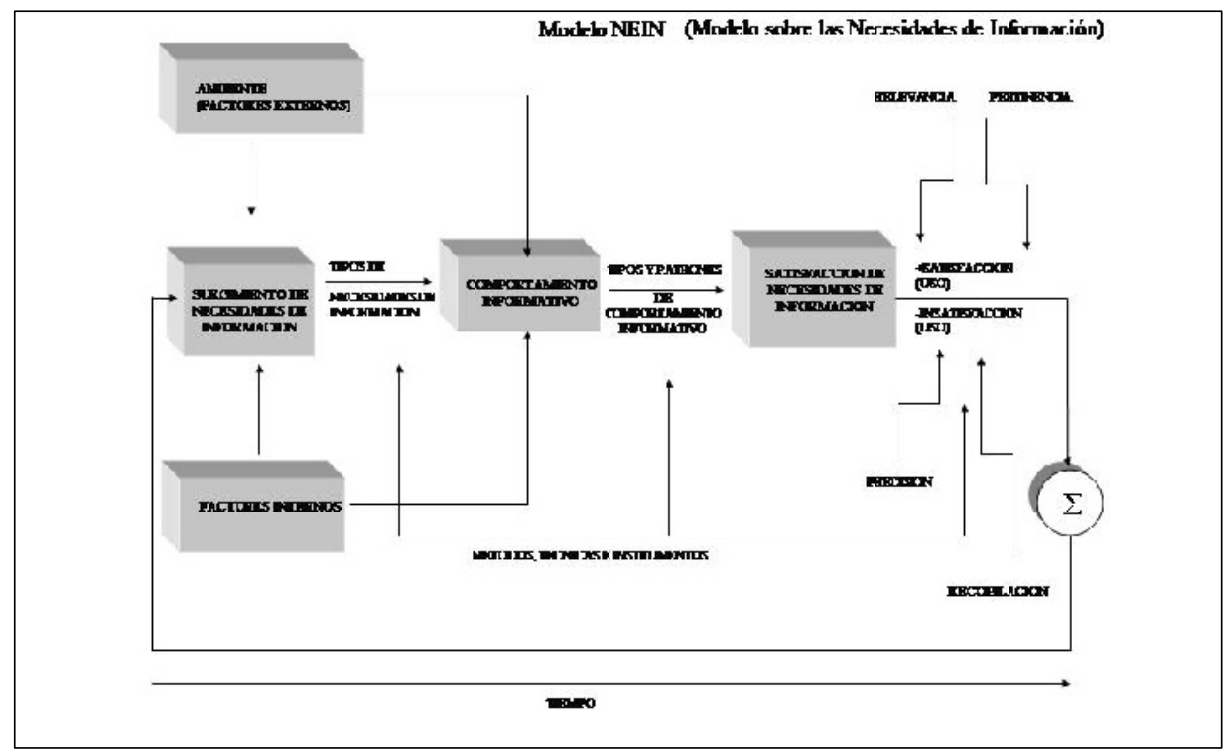

una, dos olas tres fases de este fenó meno. Véa sela siguien te figura para ubicarlas tres fasesylosfactoresexternoseinternosqueindicaelmodelo.

Pero cabe seña lar que la fase más in ves tiga da y que se pue de con si de rarla que menos dificul tadespresentaeslacorrespondientealcomportamientoenlabúsquedade in forma ción. Es de esta for ma, por lo tan to, que el nú me ro de in ves tiga cio nes en focadoaestafaseeselevadoenlaliteraturasobrelaespecialidad.

\section{LAS IN VEST IGACIONES REALIZADAS}

Eldesarrollodeinves tigacionesenlas que serelacionaalas diversascomunidades desujetos con susunidades de in forma ción fue ronllevadas a cabo demanera es porá dicaensusinicios, másbien es tu diosempíricos,y sinuna metodologíaformal.

Según Kunz los primeros estudios datan de 1920 pero entraron en auge desde 1955 y ya en 1977 exis tían mas de 2000 títu los. ${ }^{11}$ Enes tos pri me ros tra bajos se pue de ob servarquelos in ves tiga do res sólo te nían como su je to de es tu dio a los usua rios de la unidad informativa y la búsqueda de información que realizan,los materiales que consultanylosquesolicitanenpréstamo.

Desde los años cuarenta se ini cia ronlasinves tiga ciones en las que se vin cula baa los usuarios con los servicios biblio te ca rios y de in for ma ción en diver sos gru pos de científicos, tecnólogos, psicólogos, sociólogos, economistas, administradores del áreagubernamental,yotrosquesiguierondespués.

11 Kunz, Op Cit, p. 10. 


\section{InvestigacónBiblicteedógica v. 18 No. 37 julio/ diciembre de 2004}

Las primeras investigaciones de los años 1959, y de 1960 a 66 sobre comportamiento informativo son principalmente hechas por la American Psychological Association.

Desde mediados de los años sesen tala literatura de dicada ainves tigaralos usua rios de los servicios bibliotecarios y de información se desarrolló rápidamente. La mayor parte de ella sobre temas de cien cia y tec no lo gía, pero un buen nú me ro tambiénabordólascien cias so ciales, prime ro, ylashumanidades des pués, siendoestoúl ti mo en los años se ten ta. Sólo has ta años más re cien tes se ha in cursio na do enlas áreas indus tria les yde ne go cios; al gunos dees tos es tu dios fueron realizados agranescala y nocongruposreducidosomuyespecíficos.

Losobjetivosdelasinvestigacionesrealizadas sobreelcomportamiento in forma tivo (aunque les llamaban investigaciones sobre necesidades de información) y uso delainformaciónfueron: ${ }^{12}$

1. Iniciar la observación del fe nó me no acerca del uso dela in forma ción o ex presión de la necesidad informativa, o ambos.

2. Predecir acerca del uso de la información.

3. Determinar la utiliza ción delain for ma ción a tra vés de su ma ne joy ma nipula ción de las condiciones esenciales en que es usada.

Los tres puntos anteriores se pueden transformar en actividades:

1. Descripción a través de la observación del uso de la información.

2. Definición conveniente y apropiada de conceptos para describir y detallar el uso de la información.

3. Teorización de relaciones causales o cuantitativas entre el uso de la información y los factores asociados.

Hay diversasinves tiga cionesen su mayo ría ex tran je ras (has ta 1975) en que se han indagadodiversos grupos de usuarios conva rias téc nicas (cen sos, encues tas, ob servaciones) einstrumentos, comosoncuestionarios, cédulas deen trevista, diariosycédulasdeobservación. Asimismoestasinvestigaciones se siguieron replicandoporlo cual no hay aportes sustanciales, pues en muchos de los casos se dieron a conocer como resultados empíricos. En varios países no se han hecho investigaciones con profundidadymetodología. ${ }^{13}$ Casocon tra rio hasucedidoenlos países desarro lla dos dondelosaspectosmetodológicossehanextendido.

A continuaciónseenlistanlasinves tiga ciones que, paraefec tos de este documento, se reconsideraron de mayor importancia; fueron realizadas con una cobertura am plia de su je tos y sin des co no cer que exis ten otras más de este tipo y que han sido reseñadasenvariasfuentes.

12 Ben-A Liptz,Li petz Ben-A mi. "In for ma tion ne eds and uses", en:Annualrevienofinformationsaience techndogy,1970, vol. 5, p. 3.

13 F. Knets chel. "In forma tion re qui rements as a ba sis for the planning of in for ma tion ac tivities", en Problemsofinformationuserneeds edit. by AI Mikhailov. Mos cow: All Union Ins ti tut te for Scien ti fic and Te chnical In for ma tion, 1975, p. 16. 


\section{1) Hopkins University Study ${ }^{14}$}

La investigación fue realizada por la John Hopkins University en Baltimore, Maryland, Es ta dos Unidos, porungru po de psi cólo gos res pon sa bles de la mis ma y afiliadosalaAmericanPsychologicalAssociation.

Seinves tiga roncues tiones en torno al uso delain forma cióny alcom porta mien to informativo de científicos de tres áreas: ciencias físicas, ciencias de la ingeniería y cien cias so ciales. Se con tó como suje tos dein ves tiga ción a 12442 cien tíficos eingenieros.

\section{2) US Departa ment of Defense ${ }^{15}$}

Esta investigación fue realizada por el Departament of Defense de los Estados Unidosy su finalidadera en con trarel comportamien toin forma tivodelpersonal,así como susa tis fac ción con res pec to al cen tro de docu mentación de la De fen sa Nacionalydelaindustriadeladefensanorteamericana.

\section{3) NASA ${ }^{16}$}

Este es tu dio fue rea li za do en la NASA con 62 compañías o empresas vinculadas conlainvestigaciónespacialnorteamericana.Elobjetivodeeste trabajoeradefinir el comportamien toin forma tivoque seguían es tasempresasenlabúsqueda delain for mación.Seutilizóelcuestionariocomoinstrumentoparalarecoleccióndedatos.

\section{4) INFROSS Study17}

Proyecto realizado bajo la responsabilidad de la Bath UniversityenInglaterra. IN FROSS corres pon de alas siglas de In for ma tion Re que riments of So cial Scien ce y fue iniciado en 1967. Los su je tos de esta inves tiga ción fueronin vestiga do res en eláreade cien cias so ciales en Ingla terra, y les fue aplica da a 2602, que co rrespon dían a una muestra.Seutilizóelcues tionario, unaentrevis tay tambiénuntercerinstrumento que fue regis trarlas observaciones de ungruporedu cidodecientíficos so ciales del servicio ex perimentalestablecido en la Bath University y en la Bristol University.Losresultados se centraron en el uso de las referencias,índices, abstracts,catálogosdebibliotecasy bibliografías, asícomoenelusodelibrosyreferenciasqueéstostienenincluidas.

14 Véanse las tres partes de la fuen te prin ci pal en la cual se des cribela in ves ti ga ción de la Hopkins Univer sity. W.D. Garvey. Commmication: thesencedsience New York: Per ga mon Press, 1979, pp. 202-224, 256-279, 412-434.

15 NORTH AMERICAN AVIATION. Autantics Division DOD user needs study. Final technical repart Aneahe im, Cali.:DOD, 1966.2v. Estain ves tiga ción fue rea liza da por elDepar ta ment of De fen se delos Es ta dos Uni dos y su finali dad era en con trar el com por ta mien to in for ma tivo del personal, asícomo su sa tis fac ción con res pec to al cen tro de do cu men ta ción de la De fen sa Na cio nal y de la industria de la defensanorteamericana.

16 J.S. Gilmore. Thedhannd of techndogy acquisition in comecial fimsand theNASA dissemination program Denver Col.: NASA, 1966.

17 M.B.Line. "In for ma tion uses and ne eds of so cial scien tists: an ovier view ofINFROSS", en Aslib 


\section{InvestigacónBiblicteedógica v. 18 N o. 37 julio/ diciembre de 2004}

\section{5) CRUS (Center for Research on user studies) 18}

ElCen ter for Re search on UserStudies des de su crea ciónen 1976 es el en carga do de larealizacióndeinvestigacionessobreelcomportamientoinformativoeneláreade humanidades en Inglaterra. Algunos de los aspectos generales obtenidos fueron los siguientes: losestudiantes en humanidades tien den a trabajarsolos;losma te rialesusualmenteutilizados sonlos prima rios; labibliotecasecons titu yeenunimportanterecurso in forma tivo, y eluso delprés ta mointerbiblio te carioes muyin ten so porparte delos sujetos.

\section{6) Voigt's Study19}

Esta investigación fue realizada bajo la responsabilidad de Melvin Voigt entre 1958 y 1959 den tro del Pro gram me Ful brightso brela re cupe ra ción yuso dela in formación por parte de los científicos. Se utilizó como instrumento la entrevista con científicos y con los bibliotecarios del servicio de información y referencia en las áreasdequímica,físicaycienciasbiológicas.

\section{7) Otros estudio en hispa noamérica}

Alrealizaruna re visiónyanálisis delasinvestiga cionesenlistadasanteriormente, a excepción de las hispanoamericanas señaladas con el inciso 7, se puede considerar quearrojanlosiguiente:

a) Los resultados sólo abarcan, principalmente, la segunda fase del fenómeno que corresponde al comportamiento informativo.

b) Se indaga sobre las fuentes y recursos de información que utilizan las comunidades estudiadas.

c) Se centran en comunidades específicas principalmente científicas y académicas.

d) Se toma en cuenta dentro de las investigaciones expuestas a la producción científica (hay que recordar que las investigaciones están realizadas con científicos).

e) No se investigan la satisfacción ni las necesidades de información, ni se las considera como una parte integral del fenómeno de las necesidades de información.

En cambio las investigaciones realizadas en hispanoamérica, principalmente, Chile,Argentina,Brasil,EspañayMéxicopresentanotrascaracterísticas.

18 Véanse diferentes estudios produc to del CRUS como:

S. Stone, Humanities information research: proceedings of a seminar, Sheffield 1980. Sheffield: Crus, 1980; C. Corkill, Doc to ral stu dents in hu ma ni ties: a small sca le pa nel study of in formation ne eds and uses, 1976-1979. She ffield: Crus, 1981; C. Corkill, y M. Mann In for ma tion ne eds inthe humanities: two pos tal surveys. She ffield: CRUS, 1978.

19 M. J. Voigt. Saientist approades toinfomation Chi ca go: Ame ri can Li brary Asso cia tion, 1961. 
Por lo anterior se presenta con más detalle una selección de las investigaciones consideradascomoimportantesenestostrespaíses.

\section{a) Chile}

EnChile serealizólainvestigación denominada:Deteccióndenecesidades deinformacióndelacomunidadacadémicachilena, ${ }^{20}$ la cualtenía como finalidad es tablecer un diagnóstico de los recursos de información, pensando sobre todo en la infraestructura de los servicios y tomando en consideración la percepción de los usuarios - que en este caso eran los académicos de tiempo completo de las instituciones deedu ca ciónsu pe riorpertene cientes al Con sejode Rec to res-sobrelas uni dades de in forma ción, así como tam bién la percepción delos res ponsables delas unidadesin formativassobreelusoquerealizabanlosusuariosdeellas.

Se utilizóla téc ni ca de la en cues ta de bi do ala dis persióngeográ ficay ca rac te rísticasdelapoblación, asimismoseempleóunamuestraestratificadaalazar.

Debidoa que elins trumen toutilizado fue un cues tiona rio quecons tabademásde 50 preguntas seob tuvieron pocas res pues tas que cubríanlos siguientes rubros: servi cios, recursoshumanos, colecciones, infraestructura.

Esta investigación abordó el comportamiento informativo, la satisfacción (sólo en cuestionesevaluativasconrespectoalosservicios de unabibliotecaounidaddein formación) y la evaluación propiamentedicha de los serviciosbibliotecarios y de información delasunidadesinformativas, ynotocacuestionessobrenecesidadesdeinformación.

Debido a quelain ves tiga ción sein clina por la evalua ción delas uni da des dein forma cióny el com por ta mien to in for ma tivo, deja delado elfenó menodelas necesida des dein forma ción de for ma in te gral y sus re sul ta dos sonutiliza bles sólo para el mejoramientodelosserviciosdedichasunidadesinformativas.

\section{b) E spaña}

Como lo menciona Sánz ${ }^{21}$ de forma ilus tra ti va y a la vez re su mi da en una de sus obras, en Es pañase han rea liza do al gu nasinves tiga cio nes en las que sein volu craal usuario, con respecto a su comportamiento en la búsqueda de información y en el usode fuentesin formativas. Lasinves tiga cionesencen trosuniversitarios es pañdes seini cian prin cipal men te con los cien tíficos de las cien cias bio mé dicas y delas áreas deinmunología,neurocienciasyquímicadepolímeros.

20 CfrLaobra de:León Urquiza, Norma, Flo ra Piñol Gómez,y Ximena Sán chezSta fo relli. Deteción deneesidades deinformadóndlacommidadacadámicadilema San tia go de Chi le: Con se jo de Rec to res. Comisión Asesora de Bibliotecas, 1992.

21 Elau tor pre sen ta una re se ña delas in ves tiga ciones rea liza das en el cam po delos es tu dios de usuarios rea liza do en Es pa ña, por lo cual con el fin de no re pe tir el tra ba jo rea li za do eneste as pec to se pue de con fron tarla obra: Elias Sánz Ca sa do, Manualdeestudiosdausuanios Ma drid: Fun da ción Germán Sánchez Rui pé rez, 1994, pp. 62-63, 72-73. 


\section{Investigacón Biblictecoógia v. 18 N o. 37 julio/ diciembre de 2004}

DondeLópezYepes ${ }^{22}$ y Sánz recono cenlas inves tigaciones realizadas porLópez PiñeiroyTerrada ${ }^{23}$ en 1972 y 1973, comolas precur so ras de as pec tos del usua rio, ca bríaagregarque sere fierenalcomportamien to in forma tivoy noalas nece sida des de informaciónnialasatisfaccióndelusuario.

Asimismo existen otras aportaciones a este campo debidas a las investigaciones dePérezAlvarez-Ossornio ${ }^{24}$ y de Gó mez, Mén dez y Vázquez, ${ }^{25}$ quienes centraron susobservacionesenlasáreasmencionadasenelpárrafoprecedente.

También es pre cisoex ponerque en elárea de ciencias so ciales las inves tiga ciones lle va das a cabo en trelos años de 1981 y 1993, fue ron ela bo ra das porlos si guien tes aut tores:Sagredo ${ }^{26}$, Caridad, ${ }^{27}$ Méndez, Villa gray San Millán, ${ }^{28}$ Al cain y Sán chez ${ }^{29}$ así comolosde GarcíayLó pez ${ }^{30}$ esteúl timosere fierealasa tis facción deusuarios.

Cabeseñalarlaimportanciadelaintervención delCen trodeIn formacióny Docur mentaciónCientíficaenalgunasdelasinvestigacionesrealizadas.

Aunquelas investiga cionesllevadasa cabo conlos usuarios dicenconteneras pectos de necesidades de información en realidad todas tratan sobre aspectos de comportamien toin formativoenlabúsquedadein formacióndelsec tordelacomunidad es tu dia da, por lo cual sólo se re fie ren a una fase del fe nó me no (to man do como re ferenciaelModelo NEIN).

Lomismosuce deconotrasinvestiga ciones sobre evaluacióndelasa tis facciónde los usua rios en cuan to a los servi cios y al uso delOPAC, como es el caso de la obra de

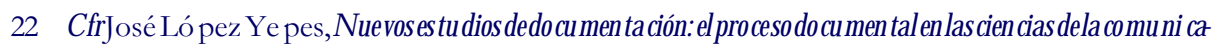
dón soial. Madrid: Ins titu to NacionaldePublicaciones, 1978

23 Cfr.J.MLó pez Piñei ro y M.L.Terrada. "Losin dica do res biblio métricos y la evalua ción de la ac tividadmédico-científica", en Medianadínica, 1992, vol. 98, pp.142-388, 384-388.

24 J.RPérezÁlvarez-Ossornio. "Estructuradelademandadein for macióndela co munidad cien tífca es pa ño la" en Reista EspañoladeDoammtacoónCientífica 1987, vol. 10, no. 1, pp. 29-44.

25 Crrlas obras de:M.Vázquez,y R. San cho, "Es tudiodelaproducción científicaespa ñolas o bre poli me ros en el pe rio do 1974-1979”, en ReistadePlásticasModamas, 1980, vol. 40, pp. 713-720.

I. Go mez, V. Cano, E. Sánzy A. Men dez. "A new applica tion of biblio me tria in di ca tos forthe assessment of research performance", en Saiere and techndogy indicators Prowedings of the first intemational workshopon sienceand techmogindicators Leiden: 1988, pp. 241-260.

26 Crr Félix Sagre do Fernán dez. "Es ta do ac tual de los ban cos de da tos en pren sa" en Ló pez Yepes, José eal. Estudiosdedbammentacóngeneraleinformativa Ma drid: Se mi na rio Mi lla res Carlo, 1981,pp. 365-377. En este do cu men to toca a los usua rios de los ban cos de da tos en pren sa.

27 Cfr.M.Caridad Sebas tián. "El usua rio on line es pañol”, en do cu men ta ción de cien cias dela in forma ción, 182, vol.6, pp.77-103.

28 Cfr. A. Mén dez, A.Villla gra y M.J. San Mi llán.Utilizadóndelasbasesdedatosautomatizadasendendiasse dalesyhumanidades ExperiendadaIISOC.Madrid:ReunióndeEspecialis tas en Centros deDocumentación, 1982.

29 Cfr.M.D. Al cain Partea rro yo yJ.M. Sán chez Nis tal.A nálisisdebibiométricoddasbúsquedasretrospectivascolliney defotodbamentaaónen psicdoǵa Ma drid: Reu nión de Es pe cia lis tas en Tele do cu men ta ción, 1982.

30 Cfr.L.A García Mele ro y M.J.Ló pez Manza ne do. "En cues ta so brelos fon dos, ca tálo gos y servicios de la Biblio te ca Na cio nal, un caso prác tico", en $\mathrm{V}$ CongesoddaAsodaacónEspañoladeA rdiveros BiblictecariosyDoammitalistas Zaragoza: ANABAD, 1991, pp. 339-355. 
Pérez Diez, ${ }^{31}$ en la que se ob serva nue va men te una ten den cia a rea li zartra bajos únicamentesobrelasegundafasedelfenómenodelasnecesidadesdeinformación.

Tomando como base el Modelo NEIN 32 se puede argumentar que las investigacio nes rea liza das y pre sen ta das en este aparta do con flu yen en la se gun da fase del fenó me no y al gu nas en la terce ra, mas no ha sido tra ta da la pri me ra de ellas que se re fiere al surgimiento de las necesidades de información, por lo cual el fenómeno en su conjuntonohasidoinvestigado.

\section{c) México}

EnMéxico serea lizó unainves tigación sobrelas ne cesi da des deinforma ciónyel comportamiento in forma tivo delosinves tigado res delaUniversidadNacional Aur tónomadeMéxico ${ }^{33}$ en todaslasáreasdelconocimiento.

Seutilizócomoins trumen to deinves tiga ciónelcues tiona rio, elcualfueenviadoa cadaunodelosinstitu tosycen tros deinves tiga cióndelauniversidad.

Su alcance se proponía detectar sus necesidades de información y su comportamiento informativo así como encontrar algunas relaciones entre diversas variables delmedioenelcuallaborabanlosinvestigadores.

Los sujetos fueron seleccionados al azar a través de una muestra estratificadade unapoblación to talde1153.

Comoresultadodelainvestiga ción sede tecta ronlas necesidades deinformación con res pec to a la tipología de au tor, so bre un asun to o proble may con cre tas. Se es tablecióunavariantedelastipologíasquepresentaFrants. ${ }^{34}$

Asimis mose encon tró que el comporta miento in forma tivoseaso cia conalgu nas variables vinculadas al área de investigación (científica, humanidades y ciencias sociales)yconeltipodenecesidaddeinformacióndetectada(sobreunautor).

Esta investigación, rea liza da en la uni ver si dad, ins ti tu ción quecuen ta con el mayor número de investigadores a nivel nacional, sólo se enfocó a dos de las fases del fenómeno, las con cernien tes a las nece sida des dein formaciónyal compor ta mien to informativodelacomunidad,perofaltóla fasedesatis faccióndelasnecesidades.

31 Cfr. Ama lia Vicen ta Pérez Diez.PerfilyniveddesatisfacoóndelosusuaniosddOPACdeunabibliotecaunivesitaria Ma drid: CINDOC, 1996.

32 Cfr Juan José Calva Gon zález. Análisis delas ne ce si da des dein forma ción do cumen tal.Teoría y mé to dos. Ma drid:JJCG, 2001. Te sis (Doc tor en Cien cias de la In for ma ción) - Uni ver si dad Complutense de Madrid.

33 Cfr.Las obras:Juan José Cal va Gon zá lez.Lasnecesidadesdeinformadóndelosinvestigadoresdláræeadeht manidadesyciendassodalesyddáreadentífica Mé xi co:UNAM, 1997. In for me de in ves tiga ción, y Juan José Cal va Gon zález, "El com por ta mien to en la bús que da de in for ma ción de los in ves tigadores delárea de hu ma ni da des y cien cias so cia les”, en Investigadónbiblidtecolóġa, 1999, vol. 13, no. 27, pp. $11-40$.

34 Va lery I. Frants. "The ne eds for in for ma tion and some as pects of in for ma tion re trie val: system cons truc tion", en:Jurmal aftheamenicansodidyforinformationsience-Vol. 39, no.2 mar. 1988. - pp. 86-91. 


\section{Investigacón Biblictecoógia v. 18 N o. 37 julio/ diciembre de 2004}

Por otrolado, los es tu dian tes de maes tría y doc to ra do del Progra made Pos gra do enBiblio te cologíay Es tu dios delaIn formación (modifica do ycrea do el doc to ra do aprobado en 1998) dentro de diversasasignaturas, han generado es tu dios so bre diversas comunidades de usuarios delain formación, algu nos deellos relativosalfenómenodelasnecesidadesdeinformación.

Entre algunas de las investigaciones realizadas, tanto publicadas como no publicadas haytesisdelicenciatura einvestigacionesgeneradasen elProgramadePosgradoenBibliotecologíayEstudiosdelaInformación,las citoacontinuación:

Álvarez González,Floridelia. Lasnecesidadesdkinformadóndelamiçindustriadealimę tosybobidasendEstadbdeY ucatán Nopublicada

AnconaMartínez,Ligia. LasnecesidadesdeinformacóndlosestudiantesdeArquitecturadla CiudaddeMéida Nopublicada.

ArtellanoJiménez,Julissa. EstudiodelasnecesidadesdeinformacóndelacommidadddCentrodeMascarones, peteneientesal CELE - UNAM Mé xi co: El au tor, 1999. Te sis (LicenciaturaenBibliotecología)-UniversidadNacionalAutónomadeMéxico.

Bel tranRodríguez, Irene. Necesidadesdinformacióndlosprofesoresinvestigadoresendárę decienaiasmaninasdedbscentrosdinnestigacón Nopublicada.

Calva González, Juan José "El comportamiento en la búsqueda de información de losin ves tiga do res del área de hu ma ni da des y cien cias so cia les", en Invetigacón biblictecoógica, 1999, vol.13, no. 27,pp.11- 40.

--. "El es tu dio de de man da: su aplica ción en las bi blio te cas", en Informacoón: produc dón, commicadónyserviaiosaño 11, número45, primavera2001.-pp.11-15.

- -. Lasnecesidadesdeinformadóndlosinvesticadoresdláreadhumanidadesyciendiassociales yddáreacientíficaMéxico: UNAM,CentroUniversitariodeInves tigaciones Bibliotecológicas,1997.Informedeinvestigación.

--. Lasnecesidadesdeinformadóndbamental. Teonáaymátodos Ma drid:JJCG, 2001. Te sis (DoctorenCienciasdelaInformación)-UniversidadComplutensedeMadrid.

- - " "Las necesidades de información del usuario en la automatización de unidades de información.” Biblicteca Univesitaria Nueva época, vol. 1, no.1, enero-junio 1998. - pp. 5-14.

--. "Las ne ce sida des dein for ma ción:impor tan cia de su es tu dio."Biblictecauniversitania, vol.9, no.3julio-septiembre, 1994.pp.33-38.

--. "Una aproxi ma ción a lo que son las nece si da des de in for ma ción”, en Investigacón biblicteedógicaMéxico:UNAM,1991, vol.5, no.11,jul.-dic.pp.33-38.

EspinalMeneses,Lilián. Necesidadesdinformadónaentíficadd CINV ESTAV end depar tamentoderearsosdlmar.Nopublicado.

García Medína, Lorena. Compartamientoinfomativodelos estudiantes deliceniatura end áreaagopeuaria Nopublicado.

García Velarde, Raúl. Necesidadesdeinformacióndelosrespansablesdebibliotecaadscitosala UnivesidadAutónomadeYucatán Nopublicado.

JiménezDávila, Rosario Gloria. Comportamientoinformativodlosdbcentesdelicenaiaturade laUniversidadPedaǵgǵcaNacianal Nopublicado. 
Ló pez Ba tes, Flor Ilea na. Comportamientainformativodestudiantesdenaestría:Antropólo gos economistasypsicálogosddaUniversidadA utónomadeY ucatán Nopublicado.

Ló pezJa ra millo, Ana Ma ría. A nálisisconceptual de téminonecesidadesdeinformadón No publicado.

- -.NecesidadesdeinformacóndelosinvestigadaresddáreadehumanidadesdlaUNAM Te sis enprocesodeMaestríaen BibliotecologíayEstudiosdelaInformación.

- -. NeesidadesycompatamientoinformativodelosinvestigadbresdeCCYDEL. México: El autor, 2000. Tesis (Licenciado en Bibliotecología)-Universidad Nacional AutónomadeMéxico.

No ve lo Pé rez, Hil da I. El compatamientoinfomativodelos estudantesdelalicendiaturaen dendiasdlacomputadóndlaunidadOrienteyFMAT.Nopublicado.

Medina Cam poy, Silvia. Laidantificacoóndenecesidadesdiinformacióndelosdbcentesdebadi lleratoendáreaformativa(crientadaresprofesionalesyvocacianales). Nopublicado.

Mén dez Ramos, Ka ren.LasnecesidadesdeinformadóndelosusuariosdlaBibliotecaddCentro delasArtesMéxico: KRM, 2003.Tesis(LicenciaturaenBibliotecología)-Universidad NacionalAutónomadeMéxico.

Mi ran da Con de, Ma ría delCarmen. Lasneecesidadesdeinformadóndelaspequeñasempresas deconencioex teriorasodadasalaCámaraNacionaldeComenciodlaciudaddeMéxico México, MCMC, 1999.Tesis(LicenciaturaenBibliotecología)-UniversidadNacional AutónomadeMéxico.

PérezFigueroa, GenaroAlberto. Elcompartamientoenlabúsquedadeinformadóndelospro pietariosdeembarcadionesribereñas:EstudiodecasoChixulubpuertoNopublicado.

RamayoLanz,Teresa. Compartamientoinformativodelosprofesoresdláreasocicoconónicasy administrativasddaUniversidadA utónomadeY ucatán Nopublica do.

RiveraEspino, Mary Carmen. Comportamientoenlabúsquedadeinformadóndelosestudian tesdlicendiaturadlaEscuelaNacionaldeMúsicaylaEsarlaSuperiordeMúsica No publicado.

RíosMartínez,JaimeJavier.Comportamientoinformativodeloses tudiantesdelter cersemestredelbachilleratodelos CCH de laUNAM.Nopublicado.

Ro mán Haza , M T.Comportamientoinformativodlosestudiantesdlalicendaturadelascame rasdequímicayfísica México: UNAM, CentroUniversitariodeInvestigaciones Bibliotecológicas, 1986.

Santos Rosas, Antonia, "Las necesidades de información de los usuarios: un estudio", enDoamentacióndeCienaiasdlaInformacón 1997,no. 20.p.207-224.

Ruz Hernán dez, Eduar do José.Necesidadesdelosusuariosquatilizanbibliotecasespedali zadasentemasyucatecosddaUniversidadA utónomadeY ucatán Nopublicado.

Santiago Pacheco, Luis Ernesto. "Necesidades y comportamiento informativo en usuarios externo de una biblioteca universitaria", en Henera. Reistadedeniasbibliotecolóġasydalainformadón Vol. 1 núm.1 ene.-jun. 2003,pp.11-36

So ria Vázquez, Juan. "La sa tis fac ción de los usua rios en la re cu pe ra ción de la in forma ción en las ba ses de da tos deSERIUNAM", en Liberrreistaddbibliotecoloǵa Nuevaépoca.México,2002,vol.4 núm.3jul.-sep.p.5-16. 


\section{Investigacón Biblictecoógia v. 18 N o. 37 julio/ diciembre de 2004}

Lasinves tiga ciones realiza das en México tan tolas publica das comolas aúnnopublicadashaninicia do suin daga ción sobrediferentessectoressocialesentreloscuales podemosmencionarlosiguiente:

Microindustria de alimentos y bebidas.

* Estudiantes de arquitectura, área agropecuaria, antropología, economía, psicología,deciencias delacomputa ción,arte, música, bachillera to, químicay física.

* Propietarios de embarcaciones ribereñas.

* Comunidades indígenas.

* Pequeñas empresas de comercio exterior.

* Docentesy/oinvestigadoresdepedagogía,bachillerato,enseñanzadelinglés y de lingǘstica aplicada, del área de ciencias marinas, humanidades, ciencias sociales y ciencias duras.

* Dictaminadores sanitarios.

Porotro lado y siguien do con el caso de Méxi co y con base en su con gre so aca démiconacionalquesonlas Jorna das MexicanasdeBiblioteconomía, enlasiguientepágina, tenemos los datos del número de documentos publicados en las memorias de este eventoyquetratanalgunadelasfases delfenómenodelasnecesidades deinformación:

Durante los 47 años de vida de las Jornadas Mexicanas de Biblioteconomía, que has ta el año an te rior sehan celebra do 34 veces, se han presentado 57 ponen cias rela tivas a este fenómeno del usua rio delain forma ción y de las necesida des in for ma tivas. En los do cu men tos re pre sen ta dos en la ta bla an te rior se veal usua rio como a un sujeto con necesidadesycomportamientoinformativo es pe cí fi cos. Aquíno se contem pla ron las que se re fie ren a otros tó pi cos que tra tan so bre elusua rio como son as pectosfilosóficos, formacióndeusuarios, reflexionessobreelusuario, etcétera.

Delas 57 po nen cias, sólo 28 se re fie ren al usua rio, ya sea en uno de sus aparta doso en toda ella, yéste es tra ta do como un su je to in fluencia do porfac to res am bien talese internos que dan origen a una tipología y que tienen que ver con sus características particulares, las que permitenla aparicióndelas necesidades dein formación. En estos documentos se contemplan: tipos, características individuales, actividades que realizan, así como aque llas po nen cias que men cio nan en una de sus par tes laim portancia de los usuarios para la administración, el diseño de servicios, el personal, los presupuestosyeledificio.

Con res pectoalas ponen cias presenta das so bre as pectos rela cio nados conel surgimiento de las necesidades de información, las cuales tocan sólo parcialmente, les corresponden 7 documentos delos 57 expuestosenlasJornadas. 
La investigación sobre las necesidades de información ... 37

\begin{tabular}{|c|c|c|c|c|c|c|}
\hline $\begin{array}{l}\text { Número } \\
\text { de } \\
\text { Jornada }\end{array}$ & Año & $\begin{array}{c}\text { Número } \\
\text { de } \\
\text { ponencias }\end{array}$ & $\begin{array}{l}\text { Sobre el } \\
\text { tema } \\
\text { usuarios en } \\
\text { general }\end{array}$ & $\begin{array}{c}\text { Sobre las } \\
\text { Necesida- } \\
\text { des de } \\
\text { información }\end{array}$ & $\begin{array}{c}\text { Sobre el } \\
\text { comporta- } \\
\text { miento } \\
\text { informativo }\end{array}$ & $\begin{array}{c}\text { Sobre la } \\
\text { Satisfacción } \\
\text { S. }\end{array}$ \\
\hline $\mathrm{I}$ & 1956 & & & & & \\
\hline II, & 1959 & & 1 & & & \\
\hline III, & 1960 & & & & & \\
\hline IV, & 1965 & & & & & \\
\hline $\mathrm{V}$ & 1969 & & & & & \\
\hline VI, & 1974 & & & & & \\
\hline VII, & 1976 & & & & & \\
\hline VIII, & 1977 & & & & & 1 \\
\hline IX, & 1978 & & 2 & & & 1 \\
\hline $\mathrm{X}$, & 1979 & & & 1 & & \\
\hline $\mathrm{XI}$, & 1980 & 8 & 5 & & 2 & 1 \\
\hline XII, & 1981 & 0 & & & & \\
\hline XIII, & 1982 & 1 & 1 & & & \\
\hline XIV & 1983 & 1 & & & 1 & \\
\hline $\mathrm{XV}$ & 1984 & 0 & & & & \\
\hline XVI, & 1985 & 6 & 1 & 1 & 3 & 1 \\
\hline XVII, & 1986 & 0 & & & & \\
\hline XVIII, & 1987 & 3 & 3 & & & \\
\hline XIX, & 1988 & 1 & 1 & & & \\
\hline $\mathrm{XX}$ & 1989 & 2 & 2 & & & \\
\hline XXI, & 1990 & 3 & & 2 & 1 & \\
\hline XXII, & 1991 & 1 & 1 & & & \\
\hline XXIII, & 1992 & 1 & & & & 1 \\
\hline XXIV, & 1993 & 3 & & & 3 & \\
\hline XXV, & 1994 & 1 & 1 & & & \\
\hline XXVI, & 1995 & 0 & & & & \\
\hline XXVII, & 1996 & 3 & & & 3 & \\
\hline
\end{tabular}


38 InvestigacónBiblictedoógia v. 18 N o. 37 julio/ diciembre de 2004

\begin{tabular}{|c|c|c|c|c|c|c|}
\hline $\begin{array}{l}\text { Núm. de } \\
\text { Jornada }\end{array}$ & Año & $\begin{array}{l}\text { Número } \\
\text { de } \\
\text { ponencias }\end{array}$ & $\begin{array}{l}\text { Sobre el } \\
\text { tema } \\
\text { usuarios en } \\
\text { general }\end{array}$ & $\begin{array}{c}\text { Sobre las } \\
\text { Necesida- } \\
\text { des de } \\
\text { información }\end{array}$ & $\begin{array}{l}\text { Sobre el } \\
\text { comporta- } \\
\text { miento } \\
\text { informativo }\end{array}$ & $\begin{array}{c}\text { Sobre la } \\
\text { Satisfacción } \\
\text { S. }\end{array}$ \\
\hline XXVIII, & 1997 & 4 & 3 & & 1 & \\
\hline XXIX, & 1998 & 5 & 2 & 1 & 1 & 1 \\
\hline XXX, & 1999 & 2 & 2 & & & \\
\hline XXXI, & 2000 & $\begin{array}{c}\text { No } \\
\text { publicadas }\end{array}$ & & & & \\
\hline XXXII, & 2001 & 4 & 3 & 1 & & \\
\hline XXXIII, & 2002 & 1 & & & 1 & \\
\hline XXXIV, & 2003 & 1 & & 1 & & \\
\hline Totales:34 & & 57 & 28 & 7 & 16 & 6 \\
\hline
\end{tabular}

En relación con las ponencias expuestas sobre el comportamiento informativo, queeslasegundafasedelfenómeno, lescorresponden 16delas57presentadas.

Paralasatis facción deusuarios, correspondientealatercerafasedelfenómenode las nece sidades dein forma ción, sepre sen ta ron 6 ponen cias delas 57 , que solo to can elaspectoparcialmente.

Las XIJornadas Mexica nas de Bibliotecono míasonlasque con tienen elmayornút me ro de ponen cias de dica das al usua rio, esto se debea que pre cisa men te tie nen como tema el usua rio, peroa a sarde ello, según el análisis sólo 8 ponen cias son rela tivas al fenó meno, ya que 5 tra tan del usua rio, nin gu na so brelas ne ce si da des de in for ma ción, 2 sobre el com por ta mien to en la bús que da dein for ma cióny 1 so brela sa tis fac ción. En estasJorna das sepresenta ron 14 ponencias delas cuales 8 tratanalgunadelas partes del fenó meno. Lo cual equivale a de cirque aun que se habló del usua rioéste fue ana liza do desdeotrospuntosdevistaynodesusnecesidadesdeinformación.

Realizandounanálisisagrupandolasjorna das des delas primerasalas dé cimas, sólo hay 6 ponen cias que co rres pon den alos años de 1956 a 1979. Dela dé ci ma prime ra ala vigé si mason 22 ponen cias pre sen tadas en trelos años 1980y 1989, ydela vigé si maprimeraalastrigésimas Jornadas setienen 23 ponen cias presentadas en trelosaños 1990 y 1999. De 2000 a 2003 te ne mos 3 po nen cias más de las 57 ponen cias pre sen ta das so bre el tó pico,porloque sehan pre sen ta do 1.9 ponen cias porañode Jorna da; es de cir, siredondeamos la cifra se tendrían 2 ponencias, aproximadamente, por Jornada, aunque en realidadexisten añosenloscuales estetemanoestratado.

Cabe señalarquela mayor parte de es tasinves tiga ciones se da en fe chas pos te riores a1998. Locual pue de reflejarun crecien te in te rés en este fe nó me no delas nece sidadesdeinformación. 


\section{d) 0 tros países de $\mathrm{H}$ ispanoamérica}

En otros paí ses, en las úl timas tres dé ca das del sigloXX, se ini ció la rea liza ción de estudios o investigacionessobre el fenómeno de las necesidades de información, el resultado fueron diversos documentos. A continuación se presentan solamente algunos de ellos, ya que serealizóuna selec ción de to dos los docu men tosen con trados que se conside ra ron re pre sen ta tivos conbase en que cu brie ran al gu no delas faseso factoresdelModelo NEIN. Seencuentranordenadosporpaís:

\section{Argentina (3 documentos, de los años: - 1971-1, 1972-1, 200-1)}

Cirigliano, Gustavo F. La condudainformativa enuniversitariosargentinos Buenos Aires: UniversidaddeBuenosAires, 1971.

Ro ma nos de Tiratel, Su sana. "Necesidades, bús que da yuso delain for ma ción: revisión de la teo ría", en Informadón, ailturaysodedadRevistadilnstitutodelnvestigadones Biblictedógicas 2000, no.2, pp.9-14.

Velázquez,Pablo.LasnecesidadesdlesusuaniosdlainformacónagícolaenAméricaLatina BuenosAires:s.n.,1972.

\section{Brasil (21 documentos de los años:1978-1, 1986-2, 1990-1, 1992-1, 1994-1, 1995-3, 1996-2, 1997-4, 1998-1, 2000-3, 2001-1, 2002-1)}

Ama ral,Sueli Angélica do. "Análise do consu mi dorbra silei ro do se tor dein formacao : aspectosculturais, so ciais, psicológicosepolíticos", en PespeetivasemCiênaia dalnformaçaQ 1996, Vol.1, No.2,pp. 207-224.

Araújo, Vania. "Pesquisadedemandaporin formacaotecnológicapelose tor produtivo", en CiendiadaInformacag 1997, Vol.26,No.3,pp. 296-306.

Barros, María Helena T.C. "Estudo do usuário na formaçao da cidadania”, en Encuen tro de EDIBCIC (5: fe bre ro 2000 : Gra na da, Es paña).Laformacóndeprofesio naleseinvestigadoresddainformacoónparalasociedadddconodimiento Grana da:Universidad de Granada, Facultad de Biblioteconomía y Documentación, 2000, pp. 429-438

Bettiol,E.M. "Necees sida des dein for macao: uma revisao", en RevistadkBiblictecono miadeBrasilia Jane-junio 1990, vol.18,no.1,pp.59-60.

Borges Duarte,Luiz Otá vio. "In formaçao parane go cios naInternet:es tudo das neces sidadesin formacionais dain dus tria moveleira de minasgerais", en Pespeetivas emCiênaiadaInformaçaa 2000, vol.5, no.1,pp. 23-40

Campos, Vania Maria Correa de. "Uso e necessidades de informacao tecnológica : um diag nós ti co do se tor de la ti cínios do Es ta do de Mi nas Ge rais", en Pespeetivas emCiênaiadaInformaçaQ 1997, Vol.2,No.1,pp.37-63.

"Es tu dioda de manda dein forma cao dos usua rios da area biotec no logia"/ . Fundacion de Tecnologia Industrial, en Ciendadainfomacaa 1986 julio-decembre, vol. 15,no.2,pp.163-192. 


\section{Investigacón Biblictecoógia v. 18 N o. 37 julio/ diciembre de 2004}

"Estudo da deman dadein for maçaono sec tordegeo ciencias e tec nologia mine ral" /.Departamen to Na cionaldaProdu cao Mineral, enCiendadainformacaoJa nau rijunio1986,vol.15,no.1,pp.81-98.

Ferreira,Sueli MaraSoaresPinto. "Novos pradigmasdain formaçao enovas percepcoesdo usua rio", en CiendadaInformaçaQ 1996, Vol.25, No.2,pp. 217-223.

Figuereido, Nice Menezes de. Estudos deuso eusuarios da informmacao Brasilia, DF: IBICT, 1994.

Gattaz, Chaves Nilce. "Um novo modelo de sistemas de informaçao para instituiçoes de P \& D”'Informaçao\& Informaça 1998, Vol.3, No.1,pp. 55- 66.

Kremer,Jea nnette Margue rite. "Es tu doda co mu nida dee dos usua rios do carro-bi bliotecada UFMG em Sao Bene di to (Dis tritode SantaLuzia, MG)", en Estudbda commidadeedos usuariosdocamobibliotecada UFMG emSaoBeneelito(DistritodeSanta Luzia,MG), 1995, Vol.24,No.2,pp.221-267.

Maria Rodriguez Hermes de. "Demanda de informaçao pelo setor industrial : dois estudos no intervalo de 25 anos" Cienia da Infomagag 1997, Vol. 26, No. 3, pp. 283-289.

Melo, L.G.C. HábitoseinteressesdosusuariosdaBibliotecaCentraldaUniversidadeFederalde Pemanbura Rio de Janeiro:IBICT, 1978.

Neves,Jorge Ta deu de Ra mos. “Análi se das neces sida des e usos dein formaçao em empresasin cubadas debio tecnología de Minas Gerais",enPerspeetivasemCiêndada Infomacaq 2000, Vol. 5,No.2,pp.217-230

Passos,EdileniceJ.L.. "OProcessolegislativoe ane cessida de dein formaçao OProcessole gisla tivo e ane ces sida de dein formaçao", en ReistaGeneraldeInformacóny Doumetaaión,2002,Vol.12,No.1,pp.191-200.

RodriguesBarbosa,Ricardo."Acessoenecessidadesdeinformaçaodepro fissionais bra sileiros: um es tu do ex plo ra tó rio”, en PespeetivasemCiêndadaInformaçaQ 1997, Vol.2,No.1,pp.5-35.

Sayao, Luiz Fernando. "Modelos teóricos em ciencia da informaçao - abstraçao e métodocientífico"CiendadaInformaçaQ 2001, Vol.30,No.1,pp.82-91.

Suaiden,EmirJosé. BiblictecapúblicaeinformaçacacommidadeSao Pau lo: Glo bal, 1995.

Suaiden,EmirJosé. "Biblio teca Publicaylasnecesidades dein formacióndela comu nidad” Investicadónbiblictedoóg்a 1992, vol. 6, no.13,pp.20-29.

Tomaél, Maria Inés. "Qualidade como fatordemo tiva cao no aten dimen to de refe rencia” Infomaçao\& InfomaçaQ 1995, Vol. 0, no.0,pp. 47- 54.

\section{Costa Rica (1 documento del año: 1998-1)}

Al tieri, Mi guel Angel. "Necesi da des y nue vos en fo ques de in for ma ción paralos or ganismos no gubernamentales y los pequeños agricultores de América Latina" AIBDA,1998,vol.14 núm.2jul./dic.pp..47-57 


\section{Colombia ( 2 documentos de los años. 1986-1, 1995-1)}

FajardoTo rres,Marga rita María. Estudiodelasnecesidadeseinteresesdeinformacóndlaco muidaddddistritoddCañoLimón Bogotá:MMFT, 1995.

HerreraCortes, Ro cío. Estudioddcompartamientodlosusuariosdlasbibliotecasdelasinstituriones de eduración supeior colonbiamas, en e proceso infarmativobúsqueda. Medellín: EIB, 1986.pp.36.

\section{Cuba ( 6 documentos de los años: 1977-1, 2983-1, 1986-1, 1990-1, 1992-1, 2000-1)}

Gorkova,V.L.. "Necesidadesinformativasdelostrabajadorescientíficos"en Adivdadesddainformadónaientíficayténica 1983, vol.14 no. 1(109) mar.pp. 1-20.

Núñez Pula,I.A. "En fo que so cio-psi co ló gico de los niveles de exis ten cia de las necesidades de formación ein formación y susimplicaciones parauname to dología deestudio",en Eđisis Vol.2, Núm.3 (mar.),pp.14-18,1977

-- "Guía metodológica para el es tu dio de las nece sida des de for ma ción y dein forma ción de los usua rios o lec to res”, en Ciendasdlainformadón 1992, vol. 23, no. 2, pp.119-123.

--." "Laido nei dad comocrite rio para eva luar la sa tis fac ción delas ne ce sida des peculia res de in forma ción”, enAdualidadesdalaInformaciónCientíficayTénica, 1986, vol. 17, no. 5 (oct.),p. 69-84.

--. "Por qué re que rimos una me to do lo gía para el es tu dio delas ne ce si da des de formación e información en las organizaciones y comunidades", en Congreso Universita rio de Cien cias dela Docu mentación e2000. Ma drid: Teo ría, his to riay metodología de las Ciencias de la Documentación: (1975-2000) / ed. José López Yepes. Madrid: Universidad Complutense de Madrid, Fa cul tad de Cien cias dela Información,2000

RodríguezLuis, Ira dia. "Es tu dio delas necesidadesin forma tivas delos pro fe siona leseinves tiga do res en elSistema Na cionaldeSalud", en Adividadksdlainformadón dentíficayténica 1990, vol.1, no.150 feb.,pp.47-64.

\section{Chile (1 documento del año: 1992-1)}

LeonUrquiza,Norma,FloraPiñolGómezy XimenaSánchezSta forelli. Lasneæsidadesdeinformacióndelacommidadacadémicadilena San tiago de Chile: Con se jo de Rectores,ComisiónAsesoradeBibliotecas, 1992

España (7 documentos de los años: 1982-3, 1987-1, 1991-1, 1994-1, 1996-1)

Al cain Partea rroyo,M.D.yJ.M.Sán chezNis tal. Análisisbibliométricodlasbúsquedasre trospeetivasanlineydefotodbamentadónempsicologáa Ma drid:Reunión deEspecialistas enTeledocumentación,1982.

CaridadSebas tian, M. "Elusua rio online es pañol”, en Doaumentadóndedendiasdlain formadón 1982, vol. 6,pp.77-103. 


\section{InestigacónBiblideedógica v. 18 N o. 37 julio/ diciembre de 2004}

García Melero, L.A. y M.J. López Manzanedo. "En cues ta so bre los fon dos, ca tálogos yservi cios delaBiblio te ca Na cional, un caso prác tico", en V CongresodelaAso daaiónEspañda deArhiveros Bildicteraniosy Doamentalistas Zaragoza: ANABAD, 1991,pp.339-355.

Men dez, A., A. Villa gra y M.J. San Mi llan.Utilizacióndlasbasesdedatosautonatizadasen denaassodalesyhumanidades Experienaiadd ISOC.Madrid:ReunióndeEspecialistas enCentrosdeDocumentación, 1982.

PérezAlvarez-Ossornio,J.R. "Estructuradelademandadein formación delacomunidadcien tífica española”, en ReistaEspañdadeDoamentaciónCientífica, 1987, vol. 10, no.1,pp.29-44.

PérezDiez, Ama lia Vicenta. PerilyniveddesatisfacióndelosusuariosdaPAC deumabbictecaunivesitania Ma drid: CINDOC, FESABIUD, 1996

Sánz Ca sa do, EliasManualdeestudiosdeusuanios Madrid:Fun da ción GermánSánchez Ruipérez,1994.

Resumiendo la cantidad de trabajos publicados que tienen relación con el fenómeno de las ne ce si da des de in forma ción en cual quiera de sus fa ses, se tie nen en hispanoaméricalossiguientes:

\begin{tabular}{|l|c|l|}
\hline \multicolumn{1}{|c|}{ País } & Número & \multicolumn{1}{c|}{ Número por año } \\
\hline Argentina & $3-$ & $1971-1,1972-1,2000-1.2001-1$ \\
\hline Brasil & $21-$ & $\begin{array}{l}1978-1,1986-2,1990-1,1992-1,1994-1,1995-3,1996-2, \\
1997-1998-1,2000-3,2001-1,2002-11978-1,1986-2,\end{array}$ \\
\hline Costa Rica & $1-$ & $1998-1$ \\
\hline Colombia & $2-$ & $1986-1,1995-1$ \\
\hline Cuba & $6-$ & $1977-1,1983-1,1986-1,1990-1,1992-1,2000-1$ \\
\hline Chile & $1-$ & $1992-1$ \\
\hline México & $33-$ & $\begin{array}{l}1986-1,1991-1,1994-1,1997-2,1998-1,1999-3,2000-1, \\
2001-5,2002-15,2003-3 .\end{array}$ \\
\hline Uruguay & $1-$ & $1995-1$ \\
\hline España & $7-$ & $1982-3,1987-1,1991-1,1994-1,1996-1$ \\
\hline
\end{tabular}

Pue deob servarse en his pa noa mérica un in te rés en realizareste tipo deinves tiga ciones sobre el fenómeno de las necesidades de información.Aunque es notorioel aumentodeellasenloconcernienteaMéxicoyBrasil. 
Cabe agre gar que exis te una lí nea de in ves ti ga ción so bre usua rios de las in formaciónenelCentroUniversitariodeInvestigaciones Bibliotecológicas ${ }^{35}$ que como una institución conlain dependen ciasuficien tedelas escuelas y facultades, puedellevara cabo, con la dedicación de tiempo suficiente, labores de investigación. A partir de 1998, conla co rres pon sa bi lidad del Pos gra do en Biblio teco logía y Es tu dios dela In formación (maes tríay doc to rado) hainvolucradoa es tudian tes de pos gradoenlaboresdeinvestigación.

\section{CONSIDERACIONES SO BRE LASIN VEST I GACIO NESY SU SRE SU L T ADOS ACERCA DE SUS NECESIDADESY COMPORT AMIENTO INFORMATIVO}

Lopresenta do enlos aparta dos an teriores deeste do cumentolleva re flexionary analizarcómo se ha con for ma dolainves tiga ción acerca delfenómeno delas necesi dadesdein forma cióna partirdel trabajocon ciertas comunidades deusuarios.

La observación que puederealizarse con los resultados de diversas investigaciones lle va das a cabo en el trans cur so delas dé ca das de 1960,1970y prin ci pios de 1980 es tánen marca das en elám bi to cien tífico.yaque comose explicóan te riormen teeste tipo de usua rios eran el tema so bre elcual se in ves tiga ba; pos te rior mente, las in ves tigaciones se inclinaron hacia los científicos de las otras áreas del conocimiento: las áreasdeciencias socialesydespuéslashumanidades.

Aunque no exis te fe cha de fini da para el ini cio de las inves tigacio nes so breelcompor tamien to delos usua rios, prime ro, y delas ne ce sida des dein for ma ción, des pués, sepue den situar como primerperio do deresultados los úl timos años dela déca da de 1940.

A par tir de la II Gue rra Mun diallas de man das in forma tivas au men ta ron de tal formaquelos sistemas deinformación científicasevieronenla necesidad dedarres puesta a esos fac to res de in cremen to queal te ra ron eldesarrollo quehas ta en ton ces ha bíanalcanzadolas bibliotecas; es tos factoressepuedenseñalarenlossiguientesincisos: ${ }^{36}$

a) Se incrementó la actividad científica.

b) Aparecieron nuevas especialidades y disciplinas.

c) Las demandas respondían al uso del conocimiento y al desarrollo del mismo.

d) Las demandas eran de cobertura internacional.

e) La formación de equipos de investigación y el procesamiento de los datos que producían, así como la necesidad de adquirir datos y conocimiento lo más pronto posible y antes que los otros grupos de investigación en el propio país o en otros.

35 Veáse el si tio web: http://cuib.unam.mx

36 H Men zel, "The in for ma tion ne eds of cu rrent scien ti fic re search", en Libraryquaterly, 1964, vol. 34, pp. 4-19 
Asíescomoelincrementodelaactividadcientíficasevereflejadoenlademandainformativayson precisa menteloscientíficosquienessecons tituyen en un tipodeusuarios delain forma ción prio rita rios fren te alos servicios biblio tecarios y dein formación y,porconsecuencia,enungrupocuyocomportamientoinformativoseráinvestigado.

Pero en sus pri me ras eta pas los re sul ta dos de las in ves tiga cio nes se ciñen a lo que Kunz ${ }^{37}$ mencio nasobrela situación delainvestigaciónempíricadelcomportamientoin formativodelosusuarios, principalmente, lacualpuedeserca racterizadapor los siguienteshechos:

1. La mayoría de las investigaciones no tienen formuladas hipótesis de trabajo precisas. Solamente investigaciones teóricas sobre estos temas pueden ser sensibles al uso de métodos para proponer una investigación práctica con los usuarios.

2. De la metodología de la investigación empírica social, la mayor parte fue desarrollada por científicos sociales y ha sido aplicada también, sin crítica alguna, en las investigaciones de las necesidades y el comportamiento informativo de los usuarios.

3. Las muestras diseñadas son frecuentemente muy pequeñas o no representativas, lo que impide llegar a generalizaciones.

4. Los métodos de análisis estadísticos frecuentemente han sido aplicados de forma inadecuada.

A raízde unare visión delasinves tiga cio nes realiza das has ta elaño de1970,Lipetz ase gu ra que si el bi blio te ca rio tie ne en cuen ta ocho de las ca rac te rís ticas delos usuarios ylas evalúa(omide) puedesa tis facerlasnece sida desdeés tos. Las caracte rís ticas quesegúnLipetzhayqueconsiderarson: ${ }^{38}$

1) Nivel funcional de lectura.

2) Nivel de satisfacción (gusto o placer derivado de la lectura).

3) Nivel de interés.

4) Nivel de capacidad (entre el material que puede leer cada vez).

5) Nivel visual (madurez física de la visión).

6) Nivel de personalidad (carácter maduro).

7) Nivel de variabilidad (rango de asimilación de varios materiales leídos).

8) Nivel de trabajo.

Hay que re sal tar que es tas ca rac te rís ticas son las que han sido es tu dia das has ta el mo mento, y no seol videquelosintere sa dos enel de sarrollo dees tasinves tigaciones delosaños60y70sonlosasociadosala AmericanPsycologicalAssociation.

37 W. Kunz, Op at, p. 19.

38 Es tos son los re sul ta dos de un análisis de va rios es tu dios re co pila dos por este au tor en un pe rio do de termina do y que es tán en la obra:LIPETZ, Ben-A mi “In forma tion needs and uses”, enAnnualre viewofinfomation siencetedhology,1970, vol. 5, pp. 3-32. 
Tam bién hay que seña lar que la ma yo ría delasin ves tiga cio nes de usua rios se han producidoa partirdelanálisis delcomporta mien toydelasdemandas dein formación de los usua rios rea les, y sólo pos te rior men te se han ex ten di do los re sul ta dos y conclu sio nes a todala po bla ción; es de cir, se ha es tu dia do pos te rior men te alos usuarios po ten cia les. Esta si tua ción hain tro du ci do un fuer te ses go en este tipo de in ves tigacio nes pues to que los usua rios rea les, como se co men tó al ini cio de este ar tí culo, represen tan unpequeñoporcentaje respec to del to talyportan to sus nece sida des ydemandasinformativasseguramentenorepresentanatodalapoblación.

En loque res pec taa las cien cias du ras tam bién se han he cho va riasinves tigaciones queabordanelcomportamientoinformativodeloscientíficos.

Según French ${ }^{39}$ el recur so queprincipalmente es utilizado porlos cien tíficos para labús que da dein forma ción son otros co le gas, es de cirotros cien tíficos den tro de su mismo tema de investigación, con lo cual queda de manifiesto que este comportamiento informativo se inscribe en la diná mica delos colegios in visiblesque exis ten en el área científica; en tan to que las fuen tes de in for ma ción que re ci ben ma yor importan ciasonlas revis tas, los libros, las me mo rias de congre sos, los abs tracts, los sistemasderecuperacióndeliteraturacientíficaylasbibliografías.

En concordancia con estos resultados Bichteler y Dederich, ${ }^{40}$ en una investigación quelle va ron a cabo congeólogos señalan quela fuenteprincipal de in forma ción deestegrupo de cien tíficos son sus pro pios cole gas, que utilizan re vis tas, preim presos, libros, catálogosbases deda tosbibliográficas ynuméricas, también tieneimportancia para estos usuarios el uso de bibliotecas y centros de documentación como medios de acceso a la búsqueda de información. También se considera que estos científicos in vier ten mu cho tiem po en la bús que da de in for ma ción que va de 2 a 10 horasporsemana.

AsimismoelDepartamentoNacionaldaProduçaoMineral ${ }^{41}$ re por ta quelos geólogosyes pe cialis tasentecnología mineral consideranimportantealabiblioteca y al centrodedocumentaciónpuesutilizansus serviciosdepréstamointerbibliotecario y de tra duc ción, pero siem pre como un lu gar para con se guir do cu men tos ya que piensanquesufuenteprincipalsiguensien doloscolegasen eláreadesuactividad.

Eneláreadebiotec nologíalaFundaçaoda Tecnologia Indus trial ${ }^{42}$ ex po ne quelos biotecnólogosutilizanlassiguien tesfuentes dein formación: preimpresos,memorias de congresos, revistas técnico científicas, estados de arte, patentes, tesis, manuales

39 A. French Beberlee. “User ne eds and library services", enLibrarytrends win ter 1990, vol. 38, no. 3, p. 419.

$40 \mathrm{~J}$ Bichtelery W.De de rich. "In for ma tion- seeking beha viour of geos cien tists", en Speial libranies Winter 1989, vol. 80, no. 3, pp. 169-178.

41 "Estudbdademandadkinformacaonosetordegeodendiaseteenologiaminera"/ .Departa men to Na cio nalda Produçao Mineral. Ciendadainformaçaa jan. jun.1986, vol.15, no. 1, pp. 81-98.

42 "Estudbdademandadkinformaçaodosusuariosdaareadekiotemologia”/ .Fun daçao da Tec no lo gia In dus trial. Ciendadainformacaa jul- dez. 1986, vol. 15, no. 2, pp. 172-175. 


\section{Investigacón Biblictecoógia v. 18 N o. 37 julio/ diciembre de 2004}

técnicos, catálogos de productos y equipos, periódicos y otras publicaciones editorialescomerciales.

Enel sectordelas humanidadesyciencias sociales,loshumanis tas ylos científicos socialestienen determinadascaracte rís ticasquelos dis tinguendeláreadelasciencias durassegúnlosresultadosdevariasinvestigaciones.

Fulton ${ }^{43}$ señala quelos hu ma nis tas trabajan de formain dividual y tienen poca conexión con sus colegas dela mis maárea, porlo cualla exis tenciade colegiosinvisibles es escasa en este grupo de sujetos. Esta visión también es compartida por Slater ${ }^{44}$ paralos cien tíficos so cia les, pero Corkill ${ }^{45}$ men cio na que es ob vio que le dan im por tan ciaal con tac to perso nal para el in tercambio deideas, y al gu nas ve ces a ma te ria les documentales.

TambiénFeliciano ${ }^{46}$ con cuerda en que porlo menos los suje tos de dica dos alas leyes no consideran que su principal fuente de información sean otros colegas y que portantosonasiduosalusodelabibliotecaocentrodedocumentación.

Porotroladolos hu manis tas y cien tí ficosso cia les tien den anodele garlas bús quedas de in formacióny se gún lo afirmaSla ter ${ }^{47}$ noutilizan in terme dia rios para hacerdichasbúsquedas. Todoparecein dicarque estecomportamien toin formativosedebe aladificul tadquerepresenta elproblematancambiante eines tabledelaterminología enlas ciencias sociales ylas hu ma ni da des, cosaquees menos fre cuente enlasciencias duras.

Con re la ción al uso de los do cu men tos y las fuen tes, se gún Ful ton ${ }^{48}$ y Sla ter, ${ }^{49}$ los humanistas tienden a hojear los documentos directamente en los estantes de la bibliote ca para des cu brirla in forma ción queles in te re sa (serendipity; pero tam bién hacenusodeotrasbibliotecasydelserviciodeprés tamointerbibliotecario.

Por su par te Agra wal ${ }^{50}$ y Case ${ }^{51}$ consideranquelos humanis tas y cien tíficossociz les tien den a usar fuen tes pri ma rias o de prime ra mano, como ma nus cri tos, libros o

43 C Fulton. "Hu ma nist as in for ma tion user: a reiew of the li te ra tu re", en Australianacademicandre searchlibranies 1991, vol. 22, no. 3, p. 189

44 M. Sla ter.Informationneelsofsodal sientists astudydeskreserchandinterviewLon don:Bri tish Li brary Re search, 1989, p. 40. Tam bién se pue de con fron tar otra obra de esta au to ra don de afir ma las mismas conclusiones:

M.Slater. "In formation needs and commu nica tion problems of so cial scien tists: the United Kingdom si tua tion", en Intemationaljaumal of infomationandlibrary rearch 1989, vol. 1, no. 2, p. 134.

45 C. Corkill y M. Mann. Informationneedsinthehumanities twopostalsurvey. She ffield: Uni ver sity of She ffield, 1978 p. 56

46 M.S. Fe li cia no. Ac ces to law: in for ma tion ne eds of re search in law and the pu blic, en Theusedthe information in thedhangingworld North Ho lland: El se vier, 1984, p. 202.

47 M. Slater, Infomation meedsofsocial scientists Op at., p. 40.

48 C. Fulton. Op at., p. 190

49 M. Sla ter. "So cial scien tists' in for ma tion ne eds in the 1980s", en Jaumal ofdbarmentation 1988, vol. 44, no. 3, p. 232.

50 S.P. Agra wal, y M. Lal. "In for ma tion ne eds of so cial scien ti fics", enIntemationallibraryreview1987, no. 19 , pp. $292-293$.

51 D.O.Case. "The co llec tion and use of in for ma tion by some ame ri can his to rians: a study of mo tives and methods", en Libraryquately. 1991, vol. 61, no.1, p. 70. 
documentos originales, tesis, reportes de investigación, informes, planes y programasgu berna mentales; mien tras que Stieg 52 ase gu ra que los his to ria do res usan los li bros, las revis tas ylos ma nus critos. Asi mis movarios au to res sos tie nen, con base en susinvestigaciones, queenlos hu manis tas predo mina eluso dela monogra fía yenlos científicos de las ciencias duras la revista; a lo que agregan que la edad de los documentos, en el caso delas hu ma nidades, noes tan relevan te comoenlasciencias du ras, yaquenopierdensuvalorconel tiempo.

Enlamayor parte delos inves tiga dores delárea dehu manida desy ciencias sociales, tambiénsetornacaracterísticoelusoencomúnyconsecutivodelosdocumentos.

Losín dices y abs tracts son poco usa dos y exis te una ma yor in clina ción aluso dela bibliografía y al servicio de compilación de bibliografías que ofrece la biblioteca o centrodedocumentación.

Comopartedelcomportamien toinformativoFulton ${ }^{53}$ y Corkill ${ }^{54}$ se ña lan quelos hu ma nis tas y cien tí fi cos sociales tien den a usar los docu men tos en gran dis per sióna tra vés de las gran des di vi sio nes del co no cimien to; es de cir, que uti li zan una gran varie dad de títulos, aun cuan do pertenez can a otrasáreas del co no cimien to, pero siempreenfuncióndeltemadeinvestigación.

De acuer do con Heim, ${ }^{55}$ los cien tíficos sociales tienen complejas necesida des deinfor ma ción por lo cual no sólo se li mi tan a ma te ria les im pre sos sino que se valen de datos dear chivo, como da tos cen sales, de vo ta cio nes y de es ta dís ticas; es de cir, in formaciónprocesadaycapturadaenbases dedatosquesonaccesiblesporcomputadora.

Otraseriedeconclusiones im portantes sedes prenden dela revisióny selecciónde investigaciones elaborada por Figueiredo ${ }^{56} \mathrm{con}$ respecto a los sectores de humanistas, cien tíficos so ciales, inves tiga do resy pro fe sores, asícomoalas pro pias unidades deinformación,yson:

1. El principio del menor esfuerzo por parte del usuario.

2. Resistencia al cambio al buscar la información en diversas fuentes y recursos.

3. La cantidad de la información necesaria para los diversos tipos de usuarios.

4. Los científicos prefieren hacer sus propias búsquedas.

5. Publicaciones profesionales y comerciales incluidas en sus sistemas de información.

6. Reducir el trabajo que se lleva a cabo en la unidad de información para entregar la información al usuario.

52 M. I. Stieg. "The information of needs of historians", en Collegeand renchlibranes November 1981, p.551.

53 Cfr. Ful ton. Op at.

54 Cfr. Corkill. Op at.

55 K.M.Heim. "So cial scien ti ficin forma tion needs for nu me ricaldata: the evolu tion of international data archive infraestructure", en Cdletionmanagement Spring 1987, vol. 9, no. 1, pp. 1-5.

56 N. M. Figueiredo. Opat,, pp. 52-61. 


\section{Investigacón Biblidtedoógica v. 18 N o. 37 julio/ diciembre de 2004}

7. Tomar en cuenta que las necesidades de información documental varían pero que al final de cuentas puede haber generalizaciones y que el sistema se adapta a esas generalidades.

8. Calidad de la información para satisfacer las necesidades del usuario.

9. La edad de los artículos de periódicos no es relevante para los humanistas.

10. Adecuar o tener los artículos que puedan necesitar los usuarios.

11. Conocimiento de los servicios de información de los humanistas.

12. Calidad de los servicios que se ofrecen a los usuarios.

13. La realización de estudios de usuarios.

14. Publicaciones extranjeras para consulta de los usuarios.

15. Comunicación oral con los usuarios.

16. Los jefes de área, sección y división o departamento de una institución deben ser entrevistados para que auxilien a determinar las necesidades de información de su personal.

17. Los estudios realizados tomando sólo la opinión y criterio de los jefes no garantizan las necesidades de información de todo el grupo de usuarios.

18. Ver los archivos, los informes que se tienen, el directorio interno de especialistas, e identificar a organizaciones técnicas y científicas.

19. Retroalimentación por parte de los usuarios.

20. Existe una dependencia muy grande de las formas orales de comunicación a través de encuentros personales y por teléfono en el comportamiento de los humanistas.

21. Dar la información con una clara identificación de los elementos de la fuente.

22. Relevancia de la información proporcionada por la unidad de información.

23. Tipos de servicios prestados.

24. Forma de brindar los servicios presencial o por medios electrónicos.

25. Cantidad de los tipos de servicios prestados.

26. Actualidad deseada de la información.

27. Rapidez en la atención a los usuarios.

28. Selectividad de la información.

29. Competencia entres las empresas que ofrecen servicios de información.

30. Facilidad de uso.

31. Identificación de la información específica.

32. Identificación del usuario con su medio ambiente y disciplina en grupos y subgrupos para corresponder al tipo de información.

33. Interacción entre bibliotecólogo y usuario - sistema/usuario.

34. La información debe ser ofrecida en formato adecuado.

35. Los registros deben ser amplios para que en ellos esté toda la información requerida y se propicie el descubrimiento accidental.

36. El sistema debe ser planeado para ofrecer cierta cantidad de información en cierto tiempo. Cantidad de información justa para satisfacer las necesidades de información. 
37. La información debe ser almacenada de tal forma que esté disponible en todo momento y sea accesible fácilmente.

38. Deben desarrollarse patrones para asegurar la utilidad de las colecciones en el futuro.

39. El sistema de información debe partir del hecho de que el usuario no puede articular sus propias necesidades de información.

40. El sistema de información debe adaptarse al comportamiento informativo del usuario y no el usuario adaptarse al sistema.

41. Debido a que el comportamiento informativo del usuario indica una tendencia hacia la comunicación oral (acudir con colegas, expertos, etcétera) el sistema de información debe crear maneras para facilitar la diseminación oral de la información.

Algo que está conformando el ambiente sobrelasinvestigacionesrelativas aeste fenómenoesqueen fechas re cien tes han empezadoaproducirseinves tiga cionessobrela pri me ra fase del mis mo; es de cir, se tra ba ja en de tec tarno sólolas ne ce si da des de información de diversos sectores de científicos y otros ámbitos no académicos sinomásbiensectores sociales deotraíndole.

Estalíneadeinvestigación realizadaporcientíficos enelDepartamentoNacional da Produçao Mineral ${ }^{57}$ logró detectar las necesidades de información de un sector for ma do porgeólogos, in ves tiga ción dela cual se des pren den las siguien tes ne ce sidadesinformativas:

* Nuevos métodos y técnicas.

Nuevos proyectos.

*stadísticas sobre reserva, producción y consumo mineral.

* Nuevos equipos y productos.

*alendario de congresos, encuentros, eventos, etcétera.

* Legislación mineral.

* Situación mineral en Brasil.

* Situación mineral en el extranjero.

* Información bibliográfica.

La Fundaçao da TecnologiaIndustrial ${ }^{58}$ también detectó las necesidadesde informaciónenelsectordelosbiotecnólogos,yarrojóalgunosdelossiguientesresultados:

* Apelaciones generales en el área de la salud.

* Detección de bacterias patógenas.

57 Es tudodademan da de in for maçao no sec tor de geo cien cias e tec nologia mine ral. Departamento Na cio nal da Pro duçao Mi ne ral, en: Ciemaa da informaçaq jan.jun.1986, vol.15, no. 1, p. 93.

58 "Es tu do da de man da de in for maçao dos usua rios da area de bio tec no lo gia" / Fun daçao da TecnologiaIndustrial. Cienia dainfomasaa jul- dez 1986, vol. 15, no. 2, p. 170. 


\section{InvestigaaónBiblictecoóġa v. 18 N o. 37 julio/ diciembre de 2004}

* Aplicaciones en la industria farmacéutica: producción de antígenos parasitarios, producción de antisueros, producción de vacunas, producción de enzimas.

* Procesos enzimáticos.

* Tecnología de la fermentación.

* Aplicaciones generales en el área agropecuaria.

* Cultura del cuidado de plantas.

* Control de plagas, bioinsecticidas.

* Mejoramiento genético vegetal.

* Nutriciónvegetal: funciónbiológicadelnitrógeno,biofertilizantes, inoculantes, micorrizas.

* Producción de vacunas (defensa de animales).

* Producción de enzimas (industria de alimentos).

* Producción de levaduras (industria de alimentos).

* Producción de proteínas unicelulares (industria de alimentos).

* Fermentación alcohólica.

* Aplicaciones generales de productos de energía: materias primas energéticas conénfa sis en ma terias amila ceas, celuloicas, sa ca rinas, residuosurbanos eindustriales, producción de etanos, producción de biogás.

* Reducción de polucionantes.

* Equipos para procesos biotecnológicos.

* Información sobre cursos de capacitación.

* Información sobre aplicaciones industriales de procesos biotecnológicos.

* Información sobre contratos de compraventa de tecnología efectuados por brasileños

* Análisis e impacto socioeconómico de la biotecnología.

* Información sobre legislación y regulaciones técnicas de interés para el área de biotecnología.

* Planesyprogramasgubernamentales cuyaimplementaciónexigeelcampode la biotecnología.

* Información sobre los servicios técnico-científicos que ofrecen biotecnología en Brasil.

* Bioinformática: programas aplicados para biotecnología.

Se puede observar claramente la diversidad de las necesidades de información que tiene este sector, de lo cual se desprende que al poseer este conocimiento las unidades de información podrán abocarse a desarrollar colecciones y servicios para sus usua rios.

Ahorabien, delos resul ta dos dees tasinvestiga ciones, asícomodelas presentadas en elapar ta do an te rior, como se pue de ob servar, exis ten di fe ren cias en cuan to a necesidadesyalcomportamientoin formativodelas diversas co munidadesanalizadas, por lo cual se puede afirmar que el medio, los factoresexternos, y las características 
de los suje tos quein te gran cada co mu nidad, asícomolos fac to res in ternos, ${ }^{59} \mathrm{in}$ fluyen en quelas necesida des y com porta mien to sean dife ren tes según se presen tenes tosfactores.

Estasinvestigaciones fueronrealizadas con diferentescomunidades deusuariosde lain for ma ción yllevadas a cabo siguien do ciertométo do, una téc nicayuninstru mento, así como una muestra de la comunidad de suje tosa es tu diar.Perotambién exis ten in da ga cio nes que no tu vie ron una se lec ción ade cua da dela mues tra, nise apli có el instrumentomásacordeconlacomunidad,nitampocohuborigorensuaplicación.

Esprecisoanalizarcuáles sonlos mé to dos que se hanutiliza do, asícomode terminar las técnicas e instrumentosempleados para poder hacer una evaluación óptima con base en la utiliza ción de es tos métodos en dife rentes comunidades de suje toso usuariosdelainformacióncautivosdealgunaunidadinformativaenparticular.

\section{CONCLUSIONES}

A través de lo presentado en los aparta dos an te rio res de este do cu men to sepuedenexponerlassiguientesreflexionesyconclusiones:

1. Las investigaciones sobre el fenómeno de las necesidades de información se están tornando importantes en América Latína y principalmente en México y Brasil, a partir de los últimos cinco años del siglo pasado y lo que va del presente, ya que se ha presentado un mayor número de investigaciones sobre diversos sectores sociales.

2. Los documentos presentados en Jornadas Mexicanas de Biblioteconomía con respecto al fenómeno de las necesidades de información son pocos, resalta la cantidad de ellos en el año 1980 (8) y durante los años de 1999 a 2003.

3. Tomando como base las tres fases del Modelo NEIN (Surgimiento de la necesidad, comportamiento informativo y satisfacción), la fase que atrae un mayor número de investigaciones es la correspondiente al comportamiento informativo. Esto deja de lado las investigaciones que se refieren al surgimiento de las necesidades de información y la identificación de éstas, y por supuesto son casi inexistentes las referidas a la satisfacción de dichas necesidades informativas.

4. Es preciso analizar las investigaciones que se han llevado a cabo así como las que se llevarán a cabo con el fin de utilizar una metodología de investigación (método, técnica e instrumento) rigurosa para obtener resultados confiables y generalizables, en poblaciones de usuarios de la información, y que permitan a la vez confirmar, modificar o rechazar los modelos teóricos que explican este fenómeno, y entonces, generar nuevo conocimiento y por tanto fundamentos teóricos.

59 Cfr. El Modelo NEIN en la obra: Juan José Calva González, Opät. 


\section{InvestigacónBiblicteedógia v. 18 N o. 37 julio/ diciembre de 2004}

5. También los resultados obtenidos de las investigaciones realizadas sobre las necesidades, comportamiento y satisfacción permiten tener elementos para el mejoramiento continuo de las unidades de información que atienden a comunidades específicas de usuarios.

6. Los estudios deberán dirigirse hacia sectores no tratados anteriormente y que no corresponden a científicos o académicos sino a sectores sociales desprotegidos como los adolescentes, los pescadores, los comerciantes, los niños, los discapacitados visuales, los estudiantes de música, etcétera.

\section{BIBLIOG RAFÍA}

AGRAWAL, S.P. y M.Lal. "In for ma tion ne eds of so cial scien ti fics", enIntemational libraryreview 1987, no. 19, pp. 292-293.

ALCAIN PARTEARROYO, M.D. y J.M. Sánchez Nistal. Análisis delabibliométricodelasbúsquedasretrospeetivasonllineydefotodbaumentaciónenpsico loǵa. Madrid: Reunión de Especialistas en Teledocumentación,1982.

BICHTELER, J y W. Dederich. "In forma tion-seekingbehaviourofgeoscientists”, en Speeial libranies Winter 1989, vol. 80, no. 3, pp. 169-178.

BRIT"TAIN, J.M. "Pitfall of user oriented research and some neglected areas", en Internationalrearchfonumininformationsiences 1981, vol.. 4, pp. 213-227.

CALVA GONZÁLEZ, Juan José. “El comporta mien to en la bús que da de información de los investigadores del área de humanidades y ciencias sociales”, en Investigacónbiblideedógica, 1999, vol. 13, no. 27, p. 11-40.

--. Lasneeridades deinformaión delosinvestigadores de área dehumanicadesy denaiassodalesyddáreadentífica Mé xico:UNAM, 1997. In for me de in vestigación.

--. Lasneecidades deinformacón doumental. Terńa ynútodos Madrid: JJCG, 2001. Te sis (Doc tor en Cien cias dela In forma ción)-Universidad Complutense de Madrid.

CARIDAD SEBASTIÁN, M. "El usua rio on line es pa ñol” Doamentacónde denias delainformadón 182, vol. 6, pp.77-103.

CASE, D.O. "The collection and use of information by some american historians: a study of motives and methods", en Libraryquately. 1991, vol. 61, no.1, p. 70 .

CORKILL, C. Dotoralstudantsinhumanitiesasmallscalepandstudyofinforma tionneets and uses, 1976-1979. Sheffield: CRUS, 1981.

CORKILL, C y M. Mann. Infomationneedsin thehumanities twopostalsurvey. Sheffield: University of Sheffield, 1978. 
CORKILL, C y M. Mann. Informationneelsinthehumanities thopostalsurveys Sheffield: CRUS, 1978.

"Estudo da de man da de in for maçao dos usua rios da area de bio tec no logia" / . Fun da cao da Tec no lo gia In dus trial, en Cienaa dainfomaça juldez. 1986, vol. 15, no. 2, pp. 172-175.

"Es tu do da de man da de in for maçao no sec tor de geo cien cias e tec nolo gia minera" / . Departa men to Na cio nalda ProducaoMine ral, enCiendada infomacao Jan.jun.1986, vol.15, no. 1, pp. 81-98.

FELICIANO, M.S. "Acces to law: information needs of research in law and the public", en Theuse of theinfomationin the hangingworld North Holland: Elsevier, 1984, p. 202.

FIGUEIREDO, N. M. Estudosdeusoeusuariosdainformacaa Brasilia : Instituto Brasileiro de Informaçao em Ciencia e Tecnologia, 1994.

FRANTS, Va lery I. "The ne eds for in for ma tion and some as pects of in formation re trie val: sys tem cons truc tion", en Jarnal oftheamericansodidyfor infomationscience1988, Vol. 39, no.2, p. 86-91.

FRENCH, BEBERLEE A. "User needs and library services", en Library trends winter 1990, vol. 38, no. 3, p. 419.

FULTON, C. "Hu ma nistas in forma tion user: a reiew of theli te ra tu re", en Australianacademicand research libranies 1991, vol. 22, no. 3, p. 189.

GARCÍA MELERO, L.A. y M.J. López Manzanedo. “Encues ta sobrelos fondos, catálogos y servicios de la BibliotecaNacional, un caso práctico",.en V Congeso dela Asociación Espeñda deArdiveros Biblicteraniosy Doumentalistas Zaragoza: ANABAD, 1991, pp. 339-355.

GARVEY, W.D. Communication: thessence of sience New York: Pergamon Press, 1979.

GILMORE, J. S. Thedhannd of techndogyacquisition in comerial fims and the NASA dissemination program Denver Col.: NASA, 1966.

GÓMEZ, I. V. Cano, E. Sanz y A. Mendez. "A new applica tion of bibliometria indicators for the assessment of research performance", en Saienceandtedhologyindicators. Proceedingsofthefirstinternational workshopon sienceand techndgy indicators Leiden: 1988, pp. 241-260.

HEIM, K.M. "Social scientific information needs for numerical data: the evolution of international data archive infraestructure", en Cdletion management Spring 1987, vol. 9, no. 1, pp. 1-5.

KNETSCHEL, F. "Information requirements as a basis for the planning ofinformationactivities", en Problensofinformationuserneeds edit. by AI Mikhailov. Moscow: All Union Institutte for Scientific and Technical Information, 1975, p. 16. 
KUNZ, W. KUNZ, W., H.W.J. Ri ttel y W. Schwu chow. Mehoos ofanalysis and evaluation of infomation neets Munchen : Verlag Dokumentation, 1977.

LEÓN URQUIZA, Norma, Flora Piñol Gómez, y Ximena Sánche Staforelli. Deteción denexsidades deinformacón dela commidad académica dilena. San tiagodeChile: ConsejodeRectores. Comisión AsesoradeBibliotecas, 1992.

LINE, M:B: "In for ma tion uses and ne eds of so cial scien tists: an ovierview of INFROSS” en Asibpromings, 1971, vol. 23, pp. 412-434.

LIPETZ,Ben-A mi. "In forma tion needs and uses", en Annualrevienofinfor mationsciencetedndogy,1970, vol. 5, p. 3-32.

LÓPEZ PIÑEIRO,J.M.yM.L.'Terrada. "Losindica do res bibliomé tricosy la evaluación de la actividad médico-científica”, en Mediana dínica, 1992, vol. 98, pp.142-388, 384-388.

LÓPEZ YEPES, José. Nuevosestudiosdedbaumentación: Aprocesodbaumentalen lasciendiasdlacommicadónsodial. Ma drid:Ins ti tu to Na cio nalde Publicaciones, 1978.

MCDIARMID, E. W. Thelibrarysurvey:problemsandmothos Chi ca go, ALA, 1940.

MÉNDEZ, A. A. Villagra y M.J. San Millan. Utilizadióndelasbasesdedatos automatizadasenciencassodalesyhumanidades Experiendadi ISOC. Ma drid: Reunión de Especialistas en Centros de Documentación, 1982.

MENZEL, H. "The information ne eds of cu rrent scien ti fic re search", en Library quately, 1964, vol. 34, pp. 4-19.

NORTH AMERICAN AVIATION. AutaneticsDivisionDOD userneedsstury. Final technical report. Aneaheim, Cali.: DOD, 1966.

PÉREZ ÁLVAREZ-OSSORNIO, J.R. "Es truc tu ra de la de man da de in forma ción de la co mu ni dad cien tífi ca es pañola”, en RevistaEspañoladeDe amentadónCientífica 1987, vol. 10, no. 1, pp. 29-44.

PÉREZ DIEZ, Amalia Vicenta. Pefil ynived desatisfación delos usuarios de OPAC deuna biblicteca univessitaria Madrid: CINDOC, 1996.

PRASAD, N.H. Infomation neel and use. Varanasi : Indian Bibliographic Center, 1992.

SAGREDOFERNÁNDEZ, Félix. "Estado actual de los bancos de datos en pren sa”, en Ló pez Ye pes, José đal. Estudiosdbdbamentadióngenerale informativa. Madrid: Seminario Millares Carlo, 1981, pp. 365-377.

SÁNZ CASADO, Elías. Manual deestudios deusuarios Madrid: Fundación Germán Sánchez Ruipérez, 1994.

SLATER, M. "So cial scien tists' in forma tion needs in the 1980 s", en Jaumal ffdoumentation 1988, vol. 44, no. 3, p. 232. 
SLATER, M. "In forma tion ne eds and commu nica tion problems of so cial scien tists: the Uni ted King dom si tua tion", en Internationaljournalofinfor mationandlibraryrearch 1989, vol. 1, no. 2, p. 134.

- -. Infomation neetsofsodal scientists astudydeskreseerchandinterview London: British Library Research, 1989, p. 40.

STIEG, M.I. "The information of needs of historians", en Cdlegeandre search libraries November 1981, p.551.

STONE, S. Humanities infomation reserch promings of a seminar, Sheffidd 1980. Sheffield: CRUS, 1980.

UNAM. CentroUniversitario de Investigaciones Bibliotecológicas [Sitio web: http:/ / cuib.unam.mx] CentroUniversitariodeInvestigaciones Biblide đớgicas México: CUIB, 2002.

VÁZQUEZ, M. Y R. Sancho. "Estudio de la producción científica española sobre polimeros en el periodo 1974-1979”, en Revista dePlásticas Modamas 1980, vol. 40, pp. 713-720.

VOIGT, M.J. Säentist' approahestoinformation Chi ca go: Ame ri can Library Association, 1961. 